\title{
COMUNICACIÓN INTEGRADA DE MARKETING: UN ACERCAMIENTO A LA EVOLUCIÓN DEL CONCEPTO*
}

\author{
Recibido: 06 de junio de 2013 • Aprobado: 15 de mayo de 2014 \\ Nelcy Rocío Escobar Moreno**
}

RESUMEN

Este documento examina el concepto de comunicaciones integradas de marketing, a través de la revisión de las diferentes conceptualizaciones y los modelos más reconocidos que lo describen. Con el objetivo de analizar el desarrollo y evolución del concepto de las comunicaciones integradas de marketing, se hace una exploración de las propuestas conceptuales que han tenido mayor impacto académico hasta la fecha, y las propuestas que lo reconocen como un concepto multidimensional.

\section{PALABRAS CLAVE}

Comunicaciones integradas de marketing, modelos de comunicación de marketing, publicidad.

\section{CLASIFICACIÓN JEL}

M31, M37

\section{CONTENIDO}

Introducción; 1. CIM: Análisis de la Evolución del Concepto; 2. Factores que inciden en el desarrollo de la CIM; 3. La Comunicación Integrada de Marketing como Concepto Multidimensional; 4. Conclusiones; Bibliografía.

\footnotetext{
Este trabajo hace parte de los productos obtenidos del trabajo de Ttsis titulada "Caracterización y evaluación preliminar de los factores determinantes de un programa de comunicaciones integradas de mercadeo para una organización no lucrativa: El caso de la Universidad Nacional de Colombia", sustentada en septiembre de 2012 para la obtención del título de M.Sc. en Administración de la Universidad Nacional, Bogotá, Colombia. Director de Tesis: Edison Jair Duque. La fase del proyecto de donde se obtiene este artículo se realizó dentro del marco de la convocatoria 2010-2011 del programa de Jóvenes Investigadores Virginia Gutiérrez de Pineda auspiciado por Departamento Administrativo de Ciencia, Tecnología e Innovación Colciencias, Colombia.

* Administradora de empresas y magíster en Administración, Universidad Nacional, Bogotá, Colombia Docente e Investigadora de la Universidad de Medellín, Colombia. Coordinadora del Grupo de Inves tigación en Mercadeo TETRIX Marketing. Dirección de correspondencia: Carrera 87 N. ${ }^{\circ}$ 30 -65, Oficina 107-bloque 5, Medellín (Antioquia), Colombia. Teléfono: +57 4 3405331. Correo electrónico: nescobar@ udem.edu.co,nrescobarm@unal.edu.co.
} 


\section{INTEGRATED MARKETING COMMUNICATIONS: A CONCEPTUAL APPROACH TO THE EVOLUTION OF THE CONCEPT}

\section{ABSTRACT}

This paper analyzes integrated marketing communications by reviewing different conceptualizations and recognized models that describe it. With the objective of analyzing the development and evolution of the integrated marketing communications concept, a brief review of the conceptual proposals that had the largest academic impacts till the moment and the proposal that recognizes it as a multidimensional concept are considered.

\section{KEY WORDS}

Integrated marketing communications, Marketing communications model, Publicity

\section{JEL CLASSIFICATION}

M31, M37

\section{CONTENT}

Introduction; 1. CIM: Conceptual evolution analysis; 2. Factors that affect the CIM development; 3. Integrated Marketing Communication as a multidimentional concept; 4. Conclusions; Bibliography.

\section{COMUNICAÇÃO INTEGRADA DE MARKETING: UMA APROXIMAÇÃO À EVOLUÇÃO DO CONCEITO}

\section{RESUMO}

Este documento examina o conceito de comunicações integradas de marketing, através da revisão das diferentes conceptualizações e os modelos mais reconhecidos do que o descrevem. Com o objetivo de analisar o desenvolvimento e evolução do conceito das comunicações integradas de marketing, realiza-se uma breve revisão das propostas conceituais que tiveram maior impacto acadêmico até a data, e as propostas que o reconhecem como um conceito multidimensional.

\section{PALAVRAS CHAVES}

Comunicações Integradas de Marketing, Modelos de Comunicação de Marketing, Publicidade.

\section{CLASSIFICAÇÃO JEL}

M31, M37.

\section{CONTEÚDO}

Introdução; 1. CIM: Análise da Evolução do Conceito; 2. Fatores que incidem no desenvolvimento da CIM; 3. A Comunicação Integrada de Marketing como Conceito Multidimensional; 4. Conclusões; Bibliografia. 


\section{INTRODUCCIÓN}

Tanto académicos como organizaciones se han referido durante mucho tiempo al uso eficaz de la información como base para la gestión efectiva de las relaciones con sus mercados. Así, el disponer de mecanismos adecuados para difundir la información fuera de la organización es un tema que ha cobrado importancia en el ámbito de la gestión. No obstante, son múltiples las consideraciones que plantean distintos grupos sobre el tema para lograr este objetivo.

Diversos investigadores destacados como Schultz (1991), Keegan, Moriarty, y Duncan (1992), Duncan y Everett (1993), Schultz (1997), Schultz y Walters, (1997), Hartley y Pickton (1999), Kotler y otros (1999), Schultz y Kitchen, (2000), Low (2000), Duncan (2002), Swain (2004), Schultz y Schultz (2004), Madhavaram, Bradrinarayanan, y Mc Donald (2005), y Kliatchko (2005) han tomado un camino común para proponer un foco sobre el cual se trabaje la comunicación. Este es el de la comunicación integrada de marketing (CIM).

De este modo, varios autores han comenzado a plantear la necesidad de considerar en las organizaciones modelos que permitan guiar la manera de tomar las decisiones de comunicación; así, todos ellos resaltan la importancia de crear marcos conceptuales y métodos que proporcionen un enfoque completo y disciplinado a este proceso de toma de decisiones, y hablan de la función de marketing como punto de partida para ello. Sus consideraciones, además, tienen en cuenta diversos factores para la construcción del concepto, pero en general podría decirse que giran sobre el mismo el objetivo: brindarle a la organización una solución efectiva e integrada a la gestión de la comunicación.

El presente trabajo tiene como objetivo principal analizar la evolución que ha tenido el concepto de CIM a lo largo del tiempo, los factores que ayudan a describirlo, y las definiciones que se han planteado para hacer referencia a este en el campo de la gestión de marketing.

Para cumplir este propósito, en este documento se hace una revisión a profundidad sobre distintos estudios, con miras a aportar una visión teórica e integrada de los principales elementos que comprenden el concepto de CIM que, a su vez, y como se verá a lo largo del texto, permiten entenderlo como una muy buena alternativa para la gestión de la comunicación en las organizaciones.

El punto de partida para la detección de fuentes bibliográficas para la revisión fue la búsqueda de diferentes revistas especializadas en el tema. La búsqueda se realizó para documentos publicados en el período comprendido entre 1991 y 2011, 
se tomaron las de los autores más significativos, y se revisaron las fuentes más relevantes con respecto al número de citaciones.

Para lograr el objetivo propuesto, el documento se estructura en cuatro secciones.

En primer lugar, se revisan los aportes más importantes desarrollados en torno a la conceptualización del constructo', los modelos existentes en la literatura y las principales líneas de investigación sobre el mismo; esto con el objetivo de plantear un marco conceptual que permita comprender la evolución del concepto. Enseguida, se propone el análisis de la CIM a partir de los factores que influyen su desarrollo en las organizaciones, de manera que se analiza la importancia que tienen las necesidades y expectativas de las audiencias para el tema a través del análisis de algunos modelos teóricos. En tercer lugar se debate la dimensión de la CIM. Así, se revisan las escalas de medición más importantes, y las diferentes propuestas que la definen como un concepto con múltiples dimensiones que, a su vez, permiten una mejor evaluación, medición, y comprensión de este. Finalmente, en la quinta sección se presentan las conclusiones y las implicaciones para la gestión que se obtienen de la revisión.

\section{CIM: ANÁLISIS DE LA EVOLUCIÓN DEL CONCEPTO}

En los últimos treinta años, el entorno de la comunicación ha evolucionado por distintos factores y razones que han propiciado notables cambios en el comportamiento de las organizaciones. Por esta razón, los factores del contexto y una proliferación de herramientas y medios de comunicación integradores, junto con la concepción sobre el marketing de relaciones, fueron elementos determinantes que hicieron surgir a finales de la década de 1980 las llamadas comunicaciones integradas de marketing -CIM-, concepto que, según Jiménez (2009) basado en Ducoffe y otros (1996), comenzó a ser reconocido y desarrollado una década después, y se considera como una de las prácticas con mayor impacto en el futuro.

El creciente cuerpo de literatura se ha ocupado de cuestiones relativas a la definición del concepto, tales como explicaciones de sus etapas (Keller, 2001) y la tipología de los mensajes, así como de cuestiones de aplicación, tales como la estructura, la responsabilidad de la organización, las actitudes hacia este nuevo enfoque, y la integración de disciplinas.

Para efectos de una mejor comprensión del texto, entiéndase el término "constructo" como cualquier término de difícil definición dentro de una teoría científica, de manera que es algo de lo que se sabe que existe, pero cuya definición es difícil o controvertida. 
Kerr y otros (2008) mencionan, además, que, como tal, la teoría de CIM, en gran medida, ha surgido a partir de observaciones de la práctica, según lo informado por una serie de investigaciones de otros académicos como Duncan y Everett (1993); Hartley y Pickton (1999); Kitchen y Schultz (1999); Low (2000), Swain (2004), entre muchos otros. Así, de acuerdo con Kerr y otros (2008), se puede decir que CIM es un concepto en constante y continua redefinición que refleja alrededor de 20 años de investigación y la práctica del concepto en sí mismo.

Sin embargo, la ambigüedad sobre las cuestiones de definición y la falta de una base teórica han generado en muchos investigadores la pregunta de si este es un concepto nuevo en absoluto o si, por el contrario, es una versión actualizada de lo que los profesionales de la comunicación siempre han tratado de hacer. Si bien algunos investigadores señalan la falta de contenido académico y de rigor, la simplificación y las soluciones prescriptivas, y el uso de la retórica como justificación en la CIM, otros afirman que esto no es raro en la gestión de muchos nuevos conceptos de marketing (Kerr y otros (2008), basados en Cornelissen y Lock (2000) y Gould, (2000)).

\subsection{CIM: Las primeras definiciones}

Ahora, para entender la evolución del concepto, que se convierte en la base de este debate, hay que partir de la definición utilizada por la Asociación Americana de Agencias de Publicidad (American Association of Advertising Agencies, AAAA, por sus siglas en Inglés) que la define como

[...] un concepto de planificación de las comunicaciones de marketing que reconoce el valor añadido de un plan integral que evalúa el papel estratégico de una variedad de disciplinas de comunicaciones, por ejemplo, publicidad, respuesta directa, promoción de ventas y relaciones públicas-y que combina estas disciplinas para brindar claridad, coherencia y comunicaciones de máximo impacto (Duncan y Everett, 1993, p. 31).

Esta primera definición resulta fundamental en la medida que fue la base de la cual se generó el interés por desarrollar un concepto que recogiera los fundamentos de una nueva visión de uno los alcances del marketing: la gestión de la comunicación.

De acuerdo con Kerr y otros, (2008), esta definición de la AAAA (citada por Duncan y Everett, (1993)), que fue desarrollada en colaboración con la Universidad de Northwestern, sigue utilizándose en la actualidad, aunque se centre principalmente en el proceso de CIM y en la integración táctica de las cuatro disciplinas principales.

Posterior a esta definición están los aportes realizados durante la década de los noventa por Schultz (1991), Keegan, Moriarty, y Duncan (1992), Duncan y Everett (1993), Kotler y otros (1999), entre muchos otros, que muestran la evolución misma 
del concepto de CIM y su significancia estratégica en las organizaciones. Sin embargo, es importante ahondar en los detalles de cada una de estas definiciones, evaluar los factores que permitieron a estos autores construir el concepto que aquí se representa, y en particular, identificar hasta dónde han llegado cada uno de estos postulados, pues, tal como se verá más adelante, estás definiciones han trascendido hasta la construcción de algunos modelos sobre el concepto, que han perdurado hasta hoy.

En un principio, Schultz (1991) y sus colegas de la Universidad de Northwestern definieron CIM como el proceso de manejar todos los recursos de información de un producto o servicio a los que un consumidor o prospecto está expuesto, y que mueven al consumidor hacia la compra o a mantener su fidelidad (Duncan y Caywood, 1996).

En este mismo sentido, Duncan y Everett (1993, p.31) propusieron que este concepto consiste en la "coordinación estratégica de todos los mensajes y medios utilizados por una organización para influir en el valor de marca percibido". Su planteamiento, aunque realiza un acercamiento al tema estratégico, no propone una metodología diferente con respecto a las definiciones hasta ese momento planteadas, que permita entender la aplicación de CIM; no obstante, ayuda a comprender la necesidad de entenderla y aplicarla desde un nuevo paradigma que plantea la comunicación de marketing centrada estratégicamente en los intereses del consumidor.

En vista de lo anterior, Schultz, Tannenbaum, y Lauterbom, (1993, p. 10) reconocen la necesidad de superar el vacío estratégico del concepto hasta ahora desarrollado, y afirman que

[...] la CIM es el proceso de desarrollo e implementación de distintas formas de programas de comunicación persuasiva con clientes. Así, su objetivo es condicionar de forma directa el comportamiento del público seleccionado para las comunicaciones, convirtiéndose en un proceso que empieza con el consumidor y funciona para determinar y definir cómo se deben desarrollar los programas de comunicación persuasiva.

También, reconocen que a partir de este nuevo enfoque, debe considerarse que, en todos los contactos de la empresa o marca con un cliente, el producto o el servicio son potenciales canales de distribución para futuros mensajes.

\subsection{CIM: Un nuevo paradigma Estratégico}

De acuerdo con Duncan y Caywood (1996), la CIM se define como un nuevo paradigma estratégico, planteado en un primer momento por Schultz (1991, p. 39), 
como "el proceso de controlar o influir estratégicamente en todos los mensajes, además de fomentar el diálogo con el propósito de crear y alimentar las relaciones rentables con los clientes y otros stakeholders". Esta definición propone entonces la importancia de focalizar los esfuerzos de la comunicación en la construcción de relaciones con todos los grupos interesados en la organización, donde deberán tenerse en cuenta los efectos en la respuesta comportamental de estos, al igual que las respuestas afectivas con respecto a la organización y no solo con respecto a sus marcas.

Por lo tanto, el enfoque de CIM se transforma en algo más integrado en materia de dirección hacia todas las posibles audiencias de la organización, y no exclusivamente hacia sus consumidores y, además, en un proceso orientado a los resultados en el largo plazo, en particular cuando parte del marketing de relaciones para fomentar la lealtad de los grupos de interés

De aquí que Nowak y Phelps (1994) propusieran no un nuevo concepto, pero sí unas nuevas características para entender el constructo. Estos fueron:

1. "Una sola voz" en la comunicación de marketing: se refiere a la transmisión de una imagen basada en una estrategia única, que debe tener una temática singular para sus mensajes sin importar cuál sea el canal utilizado.

2. La integración: es la encargada de unificar e integrar a las diferentes áreas de la organización entorno a la creación de la imagen de marca.

3. La coordinación: es la sinergia de las herramientas de marketing con miras a lograr la consecución del objetivo de imagen de marca, conciencia y generación de respuestas en las audiencias.

Como puede verse en esta construcción de los autores, el concepto de CIM tiene unos elementos que empiezan a darle el carácter integrado que la ha diferenciado de la comunicación de marketing convencional.

Es a partir de esto que Schultz y Schultz $(1998$, p. 13) deciden proponer una nueva definición de CIM que recoge aquellas características fundamentales identificadas hasta ahora por parte de los académicos de este tema, de manera que la definieron como "el proceso estratégico de negocio usado para planificar, desarrollar, ejecutar y evaluar programas de comunicación de marca coordinados, cuantificables, persuasivos a lo largo del tiempo con consumidores, clientes, clientes potenciales y otros públicos internos y externos relevantes". Estos autores, además, identifican esta nueva aproximación desde un análisis al que llamaron "marketing diagonal", el cual consiste en un enfoque basado en las 
tecnologías de información, y en analizar cómo las comunicaciones de marketing se dirigen desde las organizaciones hacia los consumidores. Se caracteriza por dar un enfoque de proceso de negocio, es decir, crear un sistema en la organización, basado en datos de los clientes, que permite, según ellos, que las actividades relacionadas con la comunicación se puedan planear, implementar y evaluar; además, posibilita comprender las percepciones de los clientes, y los contactos que se hacen con la marca, e impulsa un método de evaluación de las inversiones realizadas en comunicación y que involucra a los grupos de interés en general.

De acuerdo con Porcú y Barrio (2008), la característica más importante de esta definición es que por primera vez aparece el enfoque en un "proceso de negocio", un aspecto que lleva a crear sistemas en cadena a través de los cuales todas las actividades pueden ser planificadas, implementadas y evaluadas, y aún más importante, provee una metodología subyacente sobre la cual todas las decisiones de inversión en comunicación pueden ser evaluadas, puesto que se centra en una evaluación del valor potencial y actual de consumidores y clientes para la organización.

En esa misma línea, autores como Kotler y otros (1999, p.301), quienes definen CIM como "un concepto según el cual una empresa integra y cuidadosamente coordina sus variados canales de comunicación para conseguir un mensaje claro, coherente y convincente sobre la empresa y sus productos", intentan concretar una definición cercana a lo que las organizaciones empiezan a entender como un nuevo planteamiento estratégico.

Sin embargo, no todos los académicos ven la CIM como un nuevo enfoque o paradigma que traería nuevas construcciones a la teoría del marketing.

Por ejemplo, de acuerdo con Spotts y otros, (1998) esta solo "reinventa la teoría de marketing existente usando una terminología distinta"; o de acuerdo con Cornelissen y Lock (2000), la CIM se podría identificar solo como una teoría bastante superficial a través de su falta de precisiones conceptuales, falta de formalidad en su construcción, y la falta de profundidad de la investigación. Así, la visión que se tiene del concepto sería la de una moda en gestión. La idea de Cornelissen y Lock (2000) tuvo como argumento que debido a que no existen académicos o profesionales dedicados a la definición de CIM, o un reconocido sistema de medición del concepto (en lugar de evaluar la influencia y aportación de los distintos conceptos que lo componen) no puede considerarse a esta como una teoría o paradigma nuevo.

Asimismo, Percy, Rossiter y Elliot (2001, p. 36), aunque definen la CIM como la "planificación y ejecución de todos los tipos de comunicación de marketing necesarios para la marca, el servicio o la organización de cara a alcanzar unos objetivos de comunicación" (Porcú y Barrio, 2008), no reconocen en esta una nueva teoría. Ellos sostienen que, aunque 
algunos la perciben como un concepto valioso, hay una gran cantidad de evidencias que sugieren que una verdadera CIM es la excepción, y no la regla. Según estos autores, con frecuencia se considera que esta no es nada más que la utilización de varios medios para entregar un mensaje que, aunque hacen uso de una gama de diferentes herramientas de comunicación de marketing, no se identifican en conjunto como un programa (Percy, Rossiter, y Elliott, 2001).

A raíz de estos planteamientos, algunos autores decidieron aceptar el desafío y refutar aquellas observaciones en torno a la validez de CIM como teoría.

Fue de este modo como autores como Schultz y Kitchen (2000) plantearon que los argumentos hechos por Cornelissen y Lock (2000) eran basados en una versión seleccionada e incompleta de lo que en realidad era CIM, pues se habían focalizado en esta como una disciplina (es decir, bajo una categoría similar como pueden serlo las relaciones públicas o la promoción de ventas). Así, sostuvieron que el concepto debería considerarse en ese momento como un pre-paradigma en etapa de desarrollo, y por tanto, que no estaba obligado a mostrar una definición final aceptada. Sus opiniones fueron apoyadas por Gould (2000), quien consideró que una definición para la CIM debería formularse desde un importante concepto estratégico no muy diferente de otros conceptos, metodologías, o estrategias de marketing y de gestión que han surgido. Así, sostenía la importancia de no olvidar que todos estos tienen un evolutivo y discursivo comportamiento en la historia, caracterizado en que el concepto particular está definido y se redefine, a menudo, muchas veces (Kitchen, y otros, 2004).

Se ve entonces cómo después de una primera década de trabajo se empieza a contemplar en la construcción de CIM el enfoque estratégico requerido por las organizaciones para su aplicación, y el enfoque de integración que luego se sustentaría en la aplicación conjunta y afincada de herramientas de comunicación.

Autores como Shimp (2000) continuaron ese proceso de construcción y redefinición del concepto. En su caso, sugirió que aun cuando CIM pudiera definirse en una variedad de formas, cualquiera que fuesen esas definiciones se debían contemplar cinco características significativas: en primer lugar, el afectar el comportamiento de las audiencias mediante una comunicación dirigida como objetivo primordial; en segunda instancia, el cliente o la perspectiva del mismo como punto de partida del proceso de comunicación, y luego, el trabajo hacia atrás en favor de la marca; en tercer lugar, el uso por parte de la CIM de todas las formas de comunicación, y todas las fuentes de contactos de la marca y la empresa como posibles canales de distribución del mensaje; como cuarto punto, la sinergia y la coordinación con miras 
a lograr una fuerte imagen de marca; y por último, la construcción de una relación entre la marca y el cliente a través de ella.

Durante finales de la década de 1990, e inicios del siglo XXI se pudo identificar la constante preocupación de los teóricos de CIM frente a la evaluación de este enfoque desde la perspectiva financiera de la organización, pues, aun cuando elementos como los considerados por Shimp (2000) eran de gran importancia, también había que justificar la pertinencia e importancia de este nuevo paradigma para el rendimiento financiero de las organizaciones.

Fue así como, según lo citan Porcú y Barrio (2008), Schultz y Schultz (1998) introdujeron como medida de la implementación de la CIM la medición de la rentabilidad de la inversión en el cliente (ROCI, Return on Customer Investment), pues la atención se debería centrar, según su propuesta, en relacionar la inversión realizada en un cliente específico con el aumento (o la disminución) de los flujos de ingresos procedentes de ese mismo cliente. De este modo, el punto más importante es que los flujos de ingresos del cliente representen el elemento crítico de medición y no los esfuerzos individuales de comunicación.

De acuerdo con Porcú y Barrio (2008), también pueden citarse trabajos como los de Ambler, Bhattacharya, Edell, Keller, Lemon y Mittal (2002) que coinciden con Kitchen y Schultz (2001) en utilizar herramientas financieras para la medición de su impacto, tales como la ROI (Retorno sobre la Inversión), o la ROTPI (Return on Touch Point Investment), es decir, la medida de la rentabilidad de la inversión en el punto de contacto con el cliente (Schultz, Cole y Bailey (2004)); o la estimación de las mejoras en el valor de la marca y en el valor del cliente (Duncan y Mulhern, 2004; Hutton, 1996; Reid, Luxton y Mavondo, 2005, y Rust y otros, 2004).

Por su parte, Duncan (2002) propuso continuar la evaluación de CIM como nuevo paradigma del marketing a partir de la definición de esta como un proceso para gestionar las relaciones con los clientes que impulsan valor a la marca. En concreto, la definió como un proceso para crear y alimentar rentables relaciones con los clientes y otros interesados, para controlar o influir estratégicamente en todos los mensajes enviados a estos grupos y alentar el diálogo con ellos. Además, desglosa los principales elementos de su definición para ayudar a explicar su significado, y hace así aportes a la construcción de una definición más completa. Así, explica el proceso y la importancia de que todos los departamentos y agencias externas trabajen al unísono en la planificación y seguimiento de todas las fases de las relaciones establecidas desde la marca. Explica también el proceso por el cual se crean y se alimentan esas relaciones, cómo los nuevos clientes son atraídos y, cómo 
a través de la CIM se logra interactuar con ellos con el fin de encontrar maneras de satisfacer sus necesidades y deseos.

En el mismo camino, Carlson, Gove, y Dorsch (2003) indicaron que el éxito del concepto puede generar, por lo tanto, respuestas deseables de los clientes, de lo cual se puede afirmar, según Madhavaram, Badrinarayanan, y McDonald (2005), que la CIM puede hacer, de forma potencial, que las empresas sean más eficientes y efectivas en la comunicación con sus mercados objetivos previstos y, a su vez, ayudan a las empresas en el logro de un rendimiento financiero superior a través de un mayor valor de marca.

De acuerdo con esta conveniencia financiera en la ejecución correcta de los programas de CIM, también sobresale el trabajo de Schultz y Schultz (2004), quienes redefinieron el concepto y establecieron que

[... l la comunicación de marketing integrada es un proceso estratégico de negocio utilizado para planificar, desarrollar, ejecutar y evaluar programas de comunicación de marcas coordinados, medibles, persuasivos a lo largo del tiempo con consumidores, clientes, posibles clientes, empleados, socios y otros públicos objetivo externos e internos relevantes, que tiene como meta es generar tanto retornos financieros a corto plazo y construir marcas a largo plazo como valor para el accionista.

De aquí que se considere el objetivo financiero como uno de los más importantes resultados de la aplicación exitosa del mismo. En el mismo sentido, surgieron cuestiones adicionales sobre cómo medir el alcance financiero que tenía la ejecución de tales estrategias en las organizaciones, por lo cual la conceptualización se discutió bajo los requerimientos pragmáticos que tiene la evaluación de estos temas dentro de la estrategia organizacional.

De esta manera Kliatchko $(2005,11)$, tras su análisis de varias de las definiciones propuestas en la literatura, sugiere que la CIM es "el concepto y proceso de gestionar estratégicamente programas de comunicación de marcas centrados en el público, en los canales, y que conducen a unos resultados en el tiempo". Tal definición, a primera vista, podría considerarse como semejante a la que habían propuesto autores como Duncan (2002), Carlson, Gove, y Dorsch (2003), o Schultz y Schultz (2004) en años anteriores. Sin embargo, y como se verá a profundidad en el apartado referente a los factores que inciden en la CIM de este documento, Kliatchko (2005) propone una batería de elementos clave, entre ellos unos de tipo financiero, a través de los cuales podrían hacerse las mediciones respectivas frente al impacto de esta en la organización, aspecto que hace sobresalir los aportes de este autor con respecto a la nueva definición, pues no se enfoca en un solo elemento sino, como ya se mencionó, en una integración de varios de ellos. 
Ahora bien, otro aspecto dentro de la conceptualización de la CIM que también ha sido analizado por los académicos es el de la integración. Desde el planteamiento de Moriarty (1994) la diferencia general entre la postura tradicional y la integrada respecto a su efectividad se evalúa desde la situación de que un programa de comunicación integrada tiene en consideración todos los tipos de mensajes emitidos por una organización en cada punto donde un grupo de interés entra en contacto con la empresa. De aquí, según Jiménez y otros (2006) basados en Cornwell y Maignan, (1998), se puede interpretar que la efectividad de una herramienta de comunicación particular ha de valorarse de manera simultánea con los efectos del resto de herramientas, ya que todas afectan en menor o mayor grado la integridad de la comunicación de la empresa, y así es percibido por los grupos de interés.

De la misma forma, estos autores citan a Keller (2001), en su crítica al estudio de la medición de la efectividad de las comunicaciones, vista desde la integración, pues, según este último, se han dejado a un lado las interacciones potenciales entre las distintas herramientas comunicacionales elegidas por la empresa en su programa de comunicación. Por tal razón, se permite citar trabajos como los de Chang y Thorson (2004), Naik y Raman (2003), Smith y otros (2004) donde se comienza a dar un giro hacia la investigación sobre efectos sinérgicos de funciones de comunicación e interacciones entre medios. En este sentido sugieren, basados en Kitchen y otros (2004), que la integración de las comunicaciones debe reconocer no solo el valor de las funciones de comunicación por separado, sino el valor de la yuxtaposición de dichas funciones.

De acuerdo con Jiménez (2009), es de este modo como la discusión por el nivel de integración de la CIM se convirtió en una de las líneas de investigación más importantes del concepto a lo largo de su evolución. Destaca entonces importantes estudios exploratorios como los de Carlson y otros (2003); Chang y Thorson (2004), Garretson y Burton (2005), Gopalakrishna y Chatterjee (1992), Grove y otros (2007), Jin (2003-2004), Naik y Raman (2003), Navarro (2008), Schumann y otros (1996), que confirman en la práctica de las organizaciones la necesidad latente por una verdadera integración de lo que en palabras de Shimp (2003) se conoce como herramientas de CIM -publicidad, promoción de ventas, relaciones públicas, marketing directo, y todos los demás canales a través de los cuáles las organizaciones se comunican con sus audiencias-.

Valga mencionar que este aspecto no resulta ajeno a la propuesta de Kliatchko (2005) que se traía a colación con anterioridad, pues entre de los grupos de elementos que menciona como fundamentales dentro del concepto está el de múltiples canales, donde se refiere en particular a las disciplinas o herramientas de comunicación que 
permitirán a la organización reconocer los puntos de contacto y de información entre su marca y la audiencia objetivo. Asimismo, Kliatchko (2005) plantea dentro de su primera definición que serían estas audiencias objetivo ese último pilar que ayudaría a definir el constructo.

En vista de la importancia que para este análisis adquiere su modelo, se evaluará la versión actualizada del mismo en el siguiente aparte de esta revisión, donde la CIM se entiende como el concepto y el proceso de administrar estratégicamente los programas de comunicación de marca centrados en las audiencias focalizadas -lo que se conoce como stakeholders-, el contenido y los canales, y orientados a los resultados a través del tiempo. Se ve, entonces, cómo, y de acuerdo con Kerr y otros (2008), esta redefinición continua refleja poco más de dos décadas de investigación y la práctica del concepto en sí mismo.

De este modo, la definición de la que se habla hoy en día presenta una evolución notable, de manera que elementos como el concepto de audiencia no solo concibe a los consumidores sino también a todos los demás grupos de interesados en la empresa, y se habla de resultados a largo plazo tales como la construcción de marcas y el establecimiento de relaciones con ellos. Además, como se explicó, la estrategia ha sido declarada como un componente vital en casi todas las definiciones de CIM y sus aplicaciones se centran en las actividades que en un primer momento podrías identificarse como tácticas, pero que resultan siendo parte de una estrategia de comunicación transversal a toda la organización.

No obstante, la ambigüedad sobre las cuestiones de definición y la falta de una base teórica, según Kerr y otros (2008) ha causado a muchos investigadores la pregunta de si CIM es un concepto nuevo en absoluto, o si es solo una versión actualizada de lo que los profesionales de la comunicación siempre han tratado de hacer. De esta manera, basado en Cornelissen y Lock (2000), y Gould, (2000), afirma que si bien algunos investigadores señalan la falta de contenido académico y rigor de CIM, su simplificación y soluciones prescriptivas, y su uso de la retórica como justificación, otros afirman que esto no es raro en la gestión de muchos nuevos conceptos de marketing.

En este breve examen del proceso de desarrollo de la CIM como concepto, es evidente que se registran algunas dudas y recelos. Sin embargo, desde la posición trabajada en este documento, esta se ha de considerar desde lo que en palabras de Jiménez (2007) se entiende como 'el proceso estratégico de negocios' que configura un modo de gestionar la comunicación, que puede representar una capacidad en sí misma, y que, a su vez, es capaz de generar ventajas competitivas. Por tanto, y 
de acuerdo con este autor, se espera que su aplicación empresarial sea decisiva en el momento cuando las empresas planifiquen sus estrategias, con el fin último de establecer una diferenciación sostenible en el tiempo. Asimismo, se propone entenderla como una herramienta de largo alcance para velar por la sostenibilidad de las relaciones de las organizaciones con sus grupos de interés.

En la tabla 1 se presenta un compilado de las principales definiciones que han marcado la evolución del concepto de CIM, y a las cuales se ha hecho referencia hasta ahora en este documento.

Tabla 1: Definiciones de CIM

\begin{tabular}{|l|l|}
\hline \multicolumn{1}{|c|}{ Autor } & \multicolumn{1}{|c|}{ Definición } \\
\hline $\begin{array}{l}\text { Asociación Americana de Agen- } \\
\text { cias de Publicidad (AAAA) (1989) } \\
\text { (Citada por Duncan y Everett, } \\
\text { (1993)). }\end{array}$ & $\begin{array}{l}\text { Un concepto de planeación de las comunicaciones } \\
\text { programa que integra una variedad de disciplinas } \\
\text { estratégicas - por ejemplo, publicidad en general, } \\
\text { respuesta directa, promoción de ventas y relaciones } \\
\text { públicas- y combina estas disciplinas para propor- } \\
\text { cionar claridad, la coherencia y el impacto máximo } \\
\text { de la comunicación. }\end{array}$ \\
\hline Schultz (1991) & $\begin{array}{l}\text { El proceso de manejar todos los recursos de in- } \\
\text { formación de un producto o servicio a los que un } \\
\text { consumidor o prospecto está expuesto y que desde } \\
\text { el comportamiento mueven al consumidor hacia la } \\
\text { compra o a mantener su fidelidad. }\end{array}$ \\
\hline Keegan, Moriarty y Duncan (1992) & $\begin{array}{l}\text { La coordinación estratégica de todos los mensajes y } \\
\text { los medios utilizados por una organización para in- } \\
\text { fluir colectivamente en el valor percibido de su marca. }\end{array}$ \\
\hline Duncan y Everett (1993) & $\begin{array}{l}\text { La coordinación estratégica de todos los mensajes y } \\
\text { medios utilizados por una organización para influir } \\
\text { en el valor de marca percibido. }\end{array}$ \\
\hline $\begin{array}{l}\text { Schultz, Tannenbaum, y Lauter } \\
\text { bom (1993) }\end{array}$ & $\begin{array}{l}\text { El proceso de desarrollo e implementación de distin- } \\
\text { tas formas de programas de comunicación persuasiva } \\
\text { con clientes, cuyo objetivo es condicionar de forma } \\
\text { directa el comportamiento del público seleccionado } \\
\text { para las comunicaciones, convirtiéndose en un } \\
\text { proceso que empieza con el consumidor y funciona } \\
\text { para determinar y definir cómo se deben desarrollar } \\
\text { los programas de comunicación persuasiva. }\end{array}$ \\
\hline
\end{tabular}


Comunicación integrada de marketing: un acercamiento a la evolución del concepto

\begin{tabular}{|c|c|}
\hline Autor & Definición \\
\hline Nowak y Phelps (1994) & $\begin{array}{l}\text { Proceso de Comunicación basado en una estrategia } \\
\text { única de comunicación (una sola voz) la cual debe } \\
\text { tener un temática para sus mensajes sin importar } \\
\text { cuál era el canal utilizado -la publicidad, la relaciones } \\
\text { públicas y el marketing uno a uno- o el destinatario } \\
\text { final, y que es la encargada de unificar e integrar a } \\
\text { las diferentes áreas de la organización entorno a la } \\
\text { creación de la imagen de marca. }\end{array}$ \\
\hline Duncan y Caywood (1996) & $\begin{array}{l}\text { El proceso de controlar o influir estratégicamente } \\
\text { todos los mensajes, además de fomentar el diálogo } \\
\text { con el propósito de crear y alimentar las relaciones } \\
\text { rentables con los clientes y otros stakeholders. }\end{array}$ \\
\hline Schultz (1998) & $\begin{array}{l}\text { El proceso estratégico de negocio usado para pla- } \\
\text { nificar, desarrollar, ejecutar y evaluar programas de } \\
\text { comunicación de marca coordinados, cuantificables, } \\
\text { persuasivos a lo largo del tiempo con consumidores, } \\
\text { clientes, clientes potenciales y otros públicos inter- } \\
\text { nos y externos relevantes. }\end{array}$ \\
\hline Kotler y otros (1999) & $\begin{array}{l}\text { CIM es el concepto bajo el cual una empresa integra y } \\
\text { coordina cuidadosamente sus canales de comunica- } \\
\text { ción con miras a entregar un mensaje claro, coherente } \\
\text { y convincente sobre la organización y sus productos. }\end{array}$ \\
\hline Shimp (2000) & $\begin{array}{l}\text { Proceso estratégico de comunicación caracterizado } \\
\text { por afectar el comportamiento de las audiencias } \\
\text { mediante una comunicación dirigida; percibir al } \\
\text { cliente como punto de partida, utilizar todas las } \\
\text { formas de comunicación y todas las fuentes de } \\
\text { contactos de la marca y la empresa como posibles } \\
\text { canales de distribución del mensaje; tener sinergia y } \\
\text { coordinación con miras a lograr una fuerte imagen } \\
\text { de marca; y construir una relación entre la marca y } \\
\text { el cliente. }\end{array}$ \\
\hline Duncan (2002) & $\begin{array}{l}\text { Un proceso de funciones cruzadas para crear y nu- } \\
\text { trir las relaciones rentables con los clientes y otras } \\
\text { partes interesadas, para controlar estratégicamente } \\
\text { o influenciar todos los mensajes enviados a estos } \\
\text { grupos y promover el diálogo propositivo con ellos } \\
\text { a través del manejo de datos. }\end{array}$ \\
\hline
\end{tabular}




\begin{tabular}{|c|c|}
\hline Autor & Definición \\
\hline Smith y otros (2004) & $\begin{array}{l}\text { El análisis estratégico, la selección, ejecución y con- } \\
\text { trol de todos los elementos de comunicaciones de } \\
\text { marketing, que de manera eficiente (en el mejor uso } \\
\text { de los recursos), económica (costo mínimo) y eficaz } \\
\text { (el máximo de resultados) influyen en las transaccio- } \\
\text { nes entre una organización y sus clientes actuales y } \\
\text { potenciales, consumidores y clientes. }\end{array}$ \\
\hline Schultz y Schultz (2004) & $\begin{array}{l}\text { Proceso estratégico de negocio utilizado para pla- } \\
\text { nificar, desarrollar, ejecutar y evaluar programas de } \\
\text { comunicación de marcas coordinados, medibles, } \\
\text { persuasivos a lo largo del tiempo con consumidores, } \\
\text { clientes, posibles clientes, empleados, socios y otros } \\
\text { públicos objetivo externos e internos relevantes, que } \\
\text { tiene como meta es generar tanto retornos financie- } \\
\text { ros a corto plazo y construir marcas a largo plazo } \\
\text { como valor para el accionista. }\end{array}$ \\
\hline Kliatchko (2005) & $\begin{array}{l}\text { CiM es el concepto y el proceso de administrar } \\
\text { estratégicamente los programas de comunicación } \\
\text { de marca centrados en audiencias focalizadas, los } \\
\text { canales, y orientados a los resultados a través del } \\
\text { tiempo. }\end{array}$ \\
\hline Jiménez (2007) & $\begin{array}{l}\text { Proceso estratégico de negocios que configura un } \\
\text { modo de gestionar la comunicación, que puede } \\
\text { representar una capacidad en sí misma, y que a su } \\
\text { vez es capaz de generar ventajas competitivas. }\end{array}$ \\
\hline Kliatchko (2008) & $\begin{array}{l}\text { La CIM es un proceso que impulsa los negocios } \\
\text { a través de su público y un proceso de la gestión } \\
\text { estratégica de los grupos de interés, del contenido } \\
\text { del mensaje, de los canales de comunicación y de } \\
\text { los resultados de los programas de comunicación } \\
\text { de marca. }\end{array}$ \\
\hline
\end{tabular}

Fuente: elaboración propia con base en Kerr y otros (2008), Jiménez (2006), Kliatchko, (2005 y 2008)

\section{FACTORES OUE INCIDEN EN EL DESARROLLO DE LA CIM}

Ahora bien, a partir de los resultados obtenidos de este análisis de la evolución de la definición, y con el objetivo de profundizar en su estudio para una mejor comprensión de su tratamiento en las organizaciones, a continuación se hace referencia 
a los factores que influyen en su desarrollo. Así, de acuerdo con la propuesta que se plantea en esta investigación, los factores que influyen en la ejecución de la CIM en las organizaciones pueden agruparse y definirse en 1) las necesidades y expectativas de las audiencias, y 2) la Incidencia que tienen los stakeholders de la organización.

\subsection{Las necesidades y expectativas de las audiencias en la CIM}

Luego de la revisión conceptual, y su comprensión como un nuevo enfoque dentro del ámbito del marketing, se ha podido establecer la importancia que dentro de la evolución de la CIM han tenido las audiencias como receptores de lo que haga la organización. No obstante, habrá que identificar la razón por la cual las audiencias resultan importantes, y la respuesta no es otra que la misma por la cual los segmentos son importantes en el marketing general: la identificación de sus necesidades particulares.

Si se analizan con detenimiento las definiciones que se revisaron en el apartado anterior, elementos como el impacto en el consumidor y la respuesta en el comportamiento de los públicos (Schultz (1991), Keegan, Moriarty y Duncan (1992), Schultz y Schultz (1998), Smith y otros (1999), Shimp(2000), Kliatchko (2005), y Kliatchko (2008) y el fortalecimiento mismo de las relaciones y del diálogo propositivo con el cliente (en Schultz, Tannenbaum, y Lauterbom (1993); Duncan y Everett (1993), Duncan (1994), Shimp (2000), Schultz y Schultz (2004), Kliatchko (2005) y Kliatchko (2008) podría identificarse el papel que juegan los requerimientos y las necesidades de los consumidores como determinantes de la implementación de este enfoque dentro de las organizaciones.

Es por esto que a partir de este documento se propone el reconocimiento de este elemento como uno de los dos grandes factores que influyen en el desarrollo de la CIM. Pero ¿cuál es la perspectiva más integral desde la cual pueden evaluarse las necesidades de las audiencias, para obtener una mayor comprensión de su papel en el concepto?

El análisis que se ha hecho también abre la posibilidad a una respuesta: la propuesta desarrollada sobre constructo de CIM, planteada por Kliatchko (2008).

En ella se identifican unos atributos distintivos definidos como "los cuatro pilares de CIM": partes interesadas (stakeholders), el contenido, los canales, y los resultados. Desde el enfoque de la planeación del concepto se sigue un proceso, y los cuatro pilares pueden ser considerados como sus antecedentes y consecuencias.

Así, los pilares funcionan como antecedentes cuando se consideran en la planificación y ejecución de un nuevo programa de comunicación de marketing. Como 
el programa es completado dentro de su marco de tiempo previsto, el proceso incluye un mecanismo de realimentación de la medición, evaluación y análisis de las direcciones que se tomaron y garantizaron un impacto futuro y de éxito en los programas que, como se ha expuesto, se evalúan a raíz de las necesidades propias de las audiencias a las cuáles fue dirigido.

Por su parte, las mejoras, los cambios y otros ajustes derivados del análisis realizado en un programa en cualquiera de los cuatro pilares funcionan ahora como consecuencia del proceso de CIM en esta etapa. Desde su enfoque, este proceso sigue un modelo de circuito cerrado, donde los cuatro pilares pasan por un ciclo de ser a la vez los antecedentes y las consecuencias, tal y como se ilustra en la figura 1.

Figura 1: Los 4 pilares de CIM

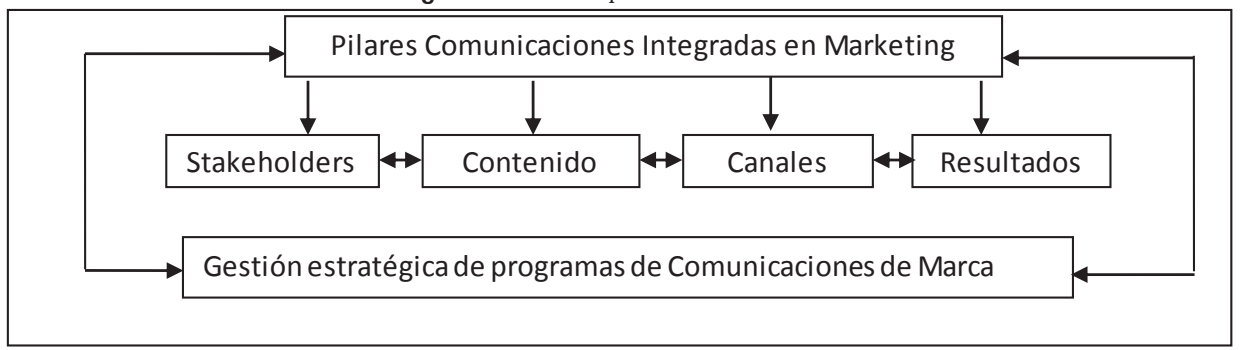

Fuente: Tomada de Kliatchko (2008, p. 145)

Lo importante de esta parte de la propuesta es que se identifica cómo Kliatchko (2008) propone, además, un listado de elementos propios para cada uno de los pilares que conforman las CIM. Estos elementos permiten vislumbrar la complejidad misma del concepto, y en particular la dimensión relacional -siempre en función de las necesidades particulares de las audiencias- que los rodea (ver figura 2).

Esta primera perspectiva permite entonces identificar un primer común denominador transversal a los 4 pilares: la relación con el cliente y sus necesidades.

Se observa, así, cómo las necesidades, los comportamientos y los valores son reconocidos en el interior del primer pilar, los stakeholders.

Tal y como lo considera Kliatchko (2008), en lugar de tener el control de los clientes o prospectos, los gerentes de CIM agers seek to be sensitive and responsive to their needs, wantstratan de ser sensibles y receptivos a sus necesidades, deseos, aspiraciones and expectations, in order to more effectively provide solutions to con- y expectativas, con el fin de proporcionar soluciones más eficaces a los sumer problems, nourish positive total customer experiences with the brandproblemas del consumidor, alimentar las experiencias positivas totales de las audiencias con 
la marca, deepen the customers' relationships with the brand and the firm, and ulti- profundizar en ellas las relaciones con la marca y la empresa, y en última instancia, mately create reciprocal value for them and the firm in the long term. crear valor recíproco para ellas y la empresa en el largo plazo.

Figura 2: Elementos de los pilares de CIM

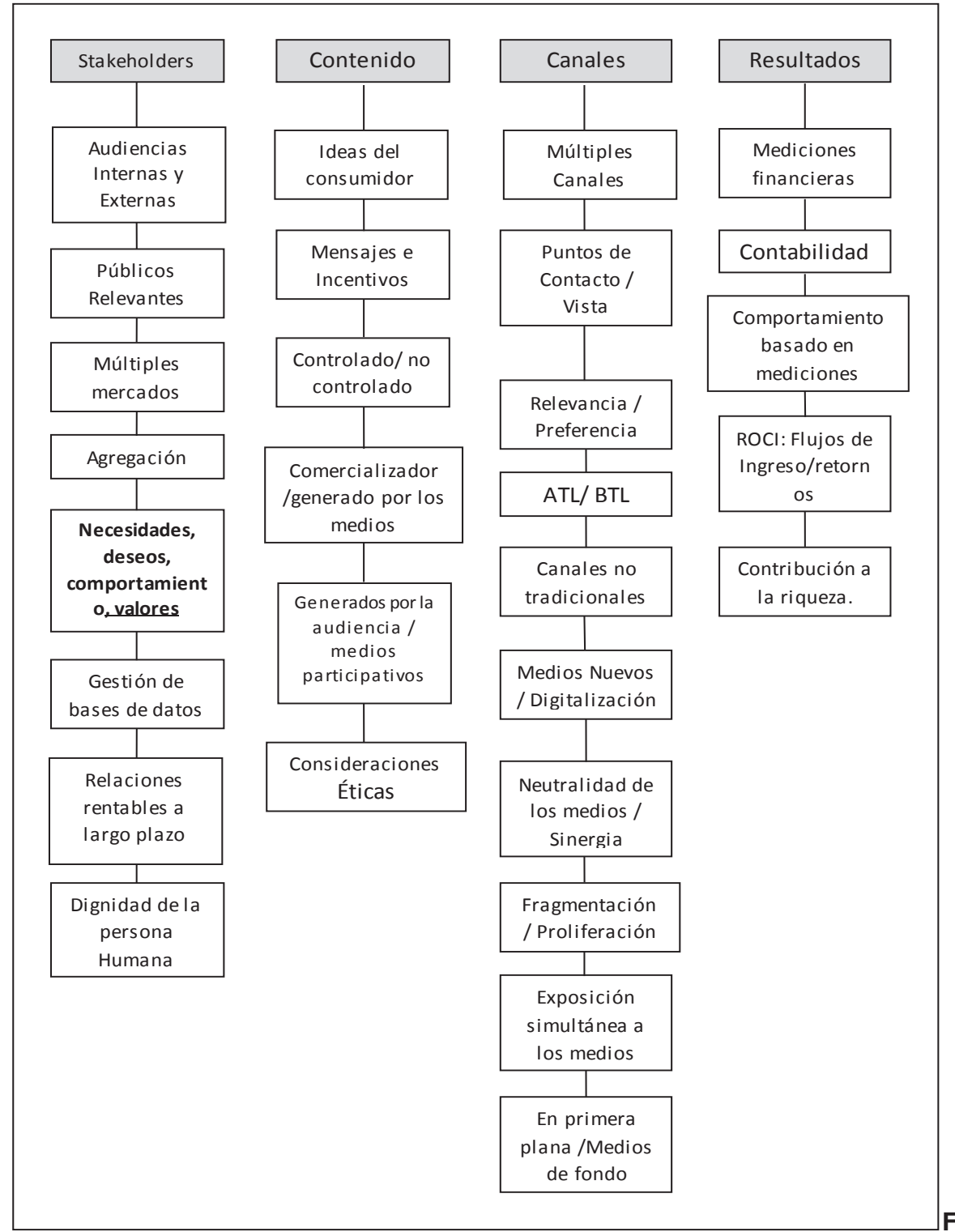

Fuente: 
Otro aspecto que refleja la incidencia de las necesidades del consumidor en las decisiones de CIM de las organizaciones se refiere a las ideas que tiene el consumidor, y su papel en el contenido mismo de las comunicaciones.

Kliatchko (2008), basado en Fortini y Campbell (1992), afirma que una apreciación de la comprensión del consumidor da lugar al entendimiento de este, y al descubrimiento de la perfecta conexión entre la marca y el cliente que en última instancia conduce a la creación de contenido atractivo.

Asimismo, en el interior del tercer pilar-los canales de comunicación-se refleja cómo una visión integrada proporciona una más amplia comprensión de los canales para incluir no solo las herramientas tradicionales -radio, televisión, medios escritos- sino todos los posibles puntos de contacto donde se encuentra el cliente o existen las perspectivas de la experiencia con respecto a una marca, garantizando el posible contacto con él de manera exitosa.

Afirma también Kliatchko (2008), con base en Schultz y Walters (1997), que con respecto a los resultados a diferencia de los modelos basados en adoptar esa actitud tradicional de medir la eficacia centrada en evaluar los efectos de la comunicación (por ejemplo, la recuperación o concienciación de la marca) y las salidas (por ejemplo, qué medios de comunicación fueron adquiridos), el enfoque CIM mide las respuestas de comportamiento (por ejemplo, las compras realizadas por clientes actuales y potenciales) y los resultados (es decir, rendimientos financieros) en términos de los flujos de ingresos de los consumidores. En el corazón del concepto, por lo tanto, se hace un proceso de valoración de los clientes, mediante la estimación del rendimiento de las inversiones (es decir, el previsto incremento de ventas logrado mediante la inversión en clientes específicos), que luego es verificada y evaluada en ciertos momentos diferentes para controlar la eficacia de los programas de CIM.

Por lo tanto, esta propuesta realizada por Kliatchko recoge esos elementos propios que promueven las necesidades de las audiencias como el punto de partida para el desarrollo de CIM, y que los académicos que han trabajado en el tema de la conceptualización han mencionado como importantes en sus definiciones sobre este nuevo enfoque.

\subsection{Los elementos del entorno y el desarrollo de CIM}

De otro lado, y de acuerdo con Schultz y Patti (2009), en la actualidad existen temas que requieren un tratamiento adicional para el desarrollo, construcción y madurez de la teoría de CIM, su investigación y práctica. 
Figura 3: Tópicos de la CiM para su desarrollo

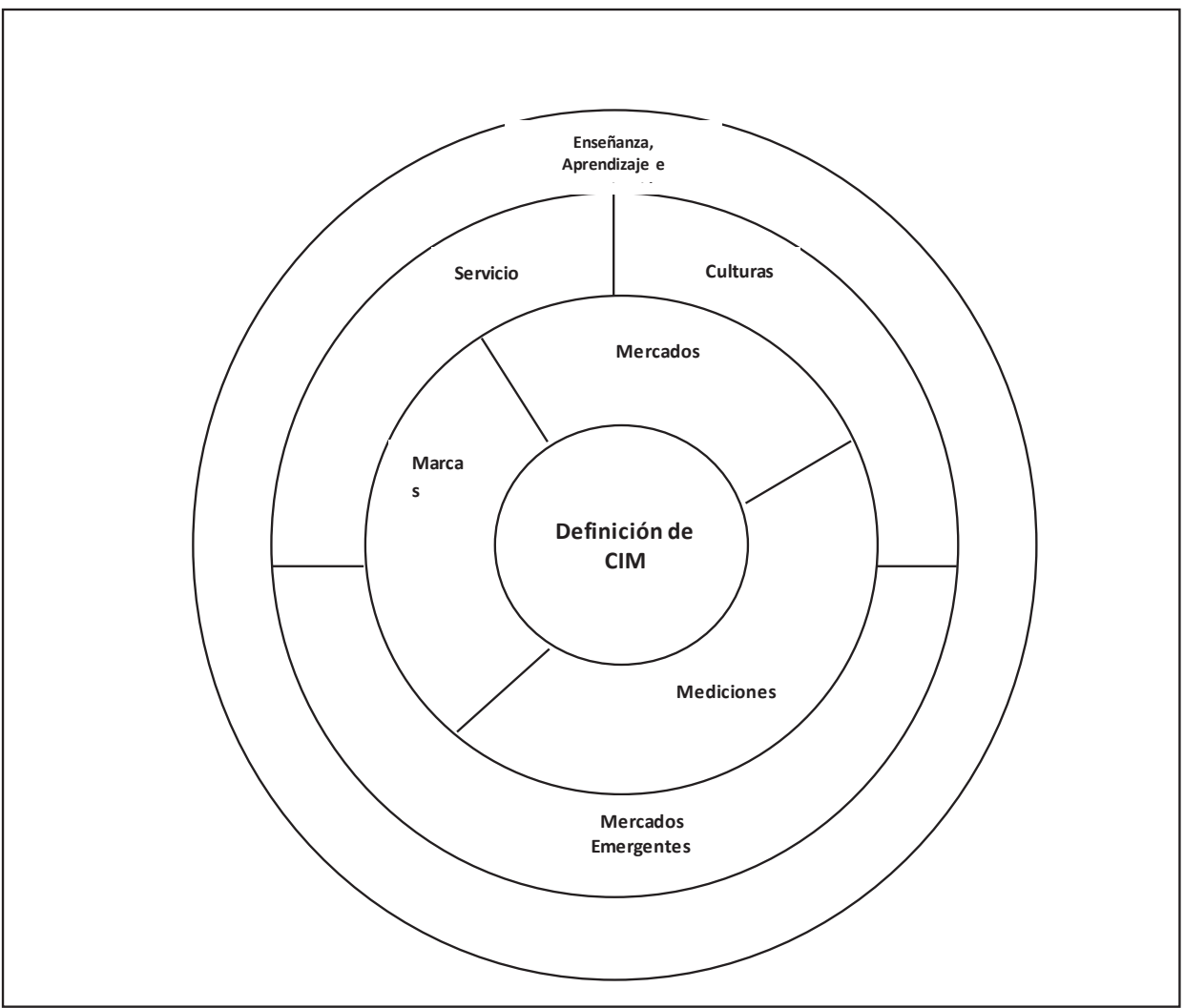

Tomada de Schultz y Patti (2009, p. 79)

Al evaluar la posición de estos autores, resulta interesante cómo los aspectos relacionados con el entorno de la organización toman gran importancia. Así, por ejemplo, el papel de los mercados y las culturas en la definición de lo que significa CIM proporciona un enfoque de trabajo hacia el estudio de las relaciones de la organización con su entorno y los otros grupos que allí se encuentran (ver figura 3).

Para entender un poco más de qué se trata este nuevo enfoque de CIM orientado bajo la incidencia de actores externos a la organización, autores como Kim, Han y Schultz (2004) han establecido que la existencia de factores socio-culturales y de circunstancias institucionales en algunos países pueden conducir a rutas de desarrollo específicas de los programas de comunicación de marketing, y que esos factores también podrían actuar como barreras o fuerzas impulsoras de la aplicación del concepto según el contexto. 
Sobre la base de esta hipótesis y de los resultados de sus investigaciones, estos autores han desarrollado un modelo sobre las rutas de desarrollo de CIM que podrían utilizarse para la investigación académica y que resultan de gran utilidad para esta revisión; pues de la misma forma que lo hace Kliatchko (2008) para el primer conjunto de factores influyentes en el desarrollo del concepto, el modelo de estos autores está alimentado por gran parte de los elementos relacionados con el entorno que, según los académicos de la CIM, pueden afectar su desarrollo en las organizaciones (p. e., Cornelissen, Lock, y Gardner (2001), Homburg y otros, 1999; Workman y otros, 1998).

Es así como este modelo propone a CIM como un proceso dinámico, donde la evolución del mercado y el ambiente de mercado son la fuerza motriz hacia la aplicación de sí mismo.

En las figuras de $4 \mathrm{a}$ y $4 \mathrm{~b}$, se ha categorizado el concepto en dos tipos según su velocidad de desarrollo. Estos se identifican en el contexto de los intereses del cliente y de las agencias de publicidad, y se rigen por factores socioculturales y de la organización. Los autores suponen que en ambos casos, el entorno de marketing favorece a la comunicación de marketing.

El escenario que se muestra en la figura 4a es la situación en la que hay más factores socioculturales e institucionales para promover la integración que los la impiden, y más factores para promover una coincidencia de intereses entre los clientes y las diferentes disciplinas de marketing que los factores que los limitan. En este caso los comunes denominadores son aplicables para que el concepto de CIM se desarrolle con bastante rapidez. Nótese, además, el papel que tienen las tecnologías de información, la segmentación de medios, y la individualización de consumidores (aspectos que se mencionaban desde la conceptualización como importantes). Por su parte, la situación que se muestra en la figura 4 b es lo contrario, y muestra que la integración debe ser aplicada con bastante lentitud.

En resumen, con respecto a los factores que influyen en el desarrollo de la CIM, se pueden establecer dos grandes grupos: las necesidades de las audiencias -explicadas bajo todos los puntos de vista trabajados desde la academia por el modelo de Kliatchko (2008)-, y los factores socioculturales que se reconocen en el entorno de la organización -explicados por los modelos de Schultz y Patti (2009), y de Kim, Han y Schultz (2004)-. Ambos grupos recogen los elementos planteados desde los aportes realizados por académicos del área en torno a su definición, y ayudan a entender el comportamiento de las organizaciones con respecto a la aplicación de este nuevo paradigma. 
Figura 4a: Ruta de desarrollo para CIM Tipo A

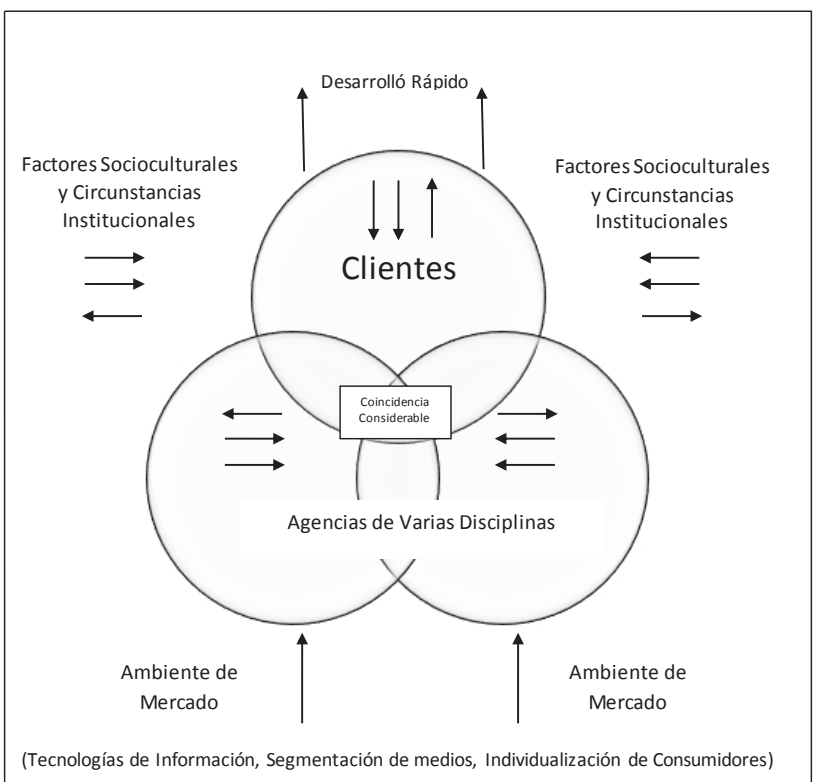

Tomada de: Kim, Han y Schultz (2004, p. 42)

Figura 4b: Ruta de desarrollo para CIM Tipo B

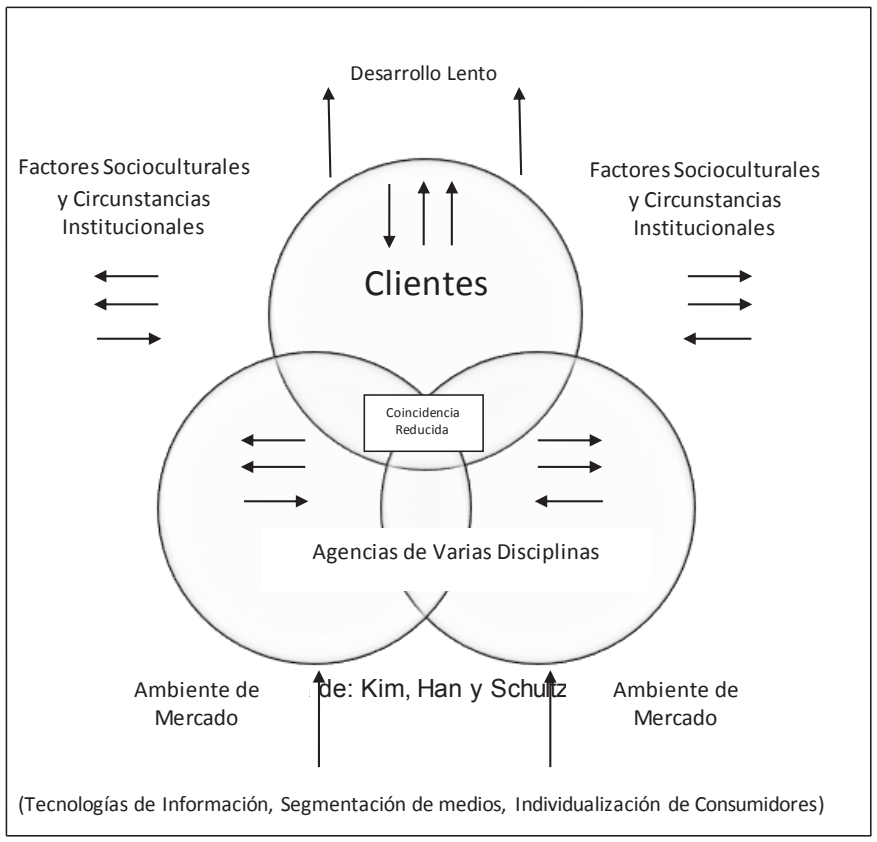

Tomada de: Kim, Han y Schultz (2004, p. 43) 
El modelo de la figura 5 ilustra la influencia descrita de estos factores.

Figura 5: Influencia de las necesidades de las audiencias y de los factores del entorno sobre la implementación de CIM en las organizaciones

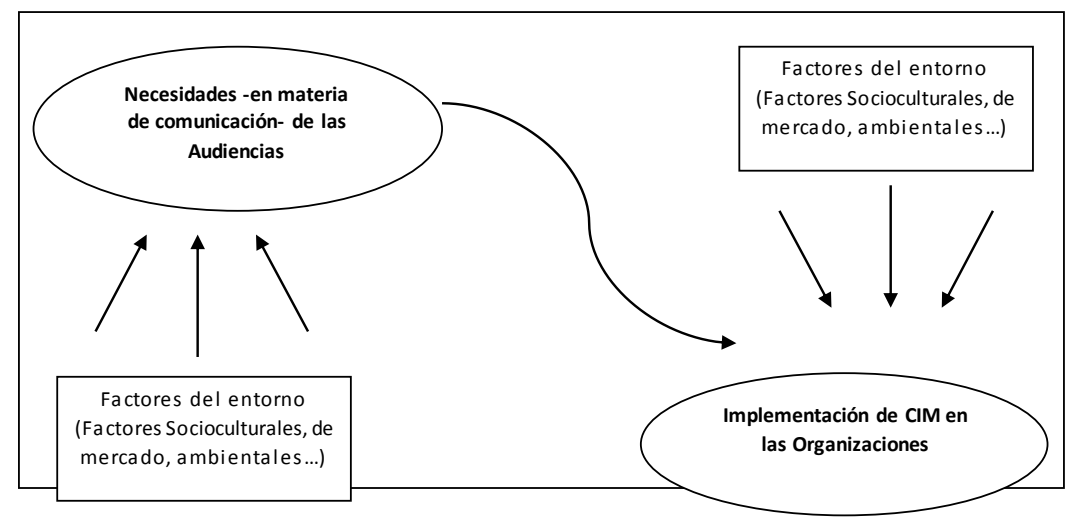

Fuente: elaboración propia

\section{LA COMUNICACIÓN INTEGRADA DE MARKETING COMO CONCEPTO MULTIDIMENSIONAL}

De acuerdo con Jiménez (2009), la escasez de propuestas teóricas sobre la dimensión del concepto CIM ha motivado que durante el proceso de desarrollo del concepto no se hayan planteado muchas alternativas frente a su medición. No obstante, desde el discurso retórico que han planteado muchos autores puede contemplarse, sin lugar a dudas, un carácter multidimensional del constructo. Es de aquí, que algunos pocos autores han hecho esfuerzos importantes en materia de generación de instrumentos de medida, validados de forma empírica para medir la CIM como un concepto multidimensional, apoyados en desarrollos conceptuales previos (p. e. Nowak y Phelps (1994), Pickton y Hartley (1998), Schultz y Schultz (1998), Kliatchko (2005)(2008), entre otros).

A continuación se exponen los más sobresalientes, y los que según el enfoque de esta investigación podrían ayudar a evaluar de una manera adecuada la implementación de esta en las organizaciones objeto de este estudio.

Para empezar, valga hacer referencia a la escala más utilizada en la literatura para medir la CIM: la escala multidimensional desarrollada por Duncan y Moriarty (1997). Este instrumento, al que se denominó "Mini-auditoría de marketing integrado", tiene como objetivo evaluar la integración de la comunicación en el interior y en el exterior de la organización. 
De acuerdo con Jiménez (2009), consiste entonces en medir la Integración de la CIM a partir de cinco dimensiones:

1. Infraestructura organizacional: Evalúa la fortaleza de las relaciones funcionales en la organización y cómo afectan la gestión de las marcas.

2. Interactividad: Evalúa los procesos que vinculan a los clientes de la empresa con sus marcas.

3. Misión de marketing: Evalúa la tenencia de una misión en la organización dirigida a la creación de valor para los grupos de interés.

4. Consistencia estratégica: Evalúa la coordinación de los mensajes y elementos del marketing mix que promocionan la marca.

5. Planificación y evaluación: Evalúa la consideración estratégica de todas las audiencias objetivo dentro de la promoción de la marca.

Reid (2005) propone una versión más reciente de esta escala, que tiene como objetivo analizar la relación entre el proceso de CIM y el desempeño de una marca en el mercado. Sin embargo presenta una pequeña modificación, pues reúne las últimas tres dimensiones en una sola, a la que le da por nombre "planificación estratégica cross-funcional".

Un segundo instrumento de gran impacto en la literatura es el diseñado por Phelps y Johnson (1996), y desarrollado a través de la técnica de análisis factorial exploratorio para datos obtenidos de una muestra de 101 empresas del sector comercial. En este, los autores señalan cinco dimensiones de la Orientación hacia la CIM; estas son: marketing directo, una única voz, campañas de comunicación de marketing coordinadas, incremento de responsabilidades, y objetivos de respuesta. De acuerdo con los autores, la principal contribución de esta escala de medida es la presentación del proceso de exploración del significado de la CIM, de manera que se contribuya al objetivo de alcanzar una definición común de ella.

Ewing, De Busy, y Caruana (2000) desarrollan una adaptación de esta escala, donde solo tienen en cuenta cuatro de las cinco dimensiones propuestas por Phelps y Johnson (1996) -no tienen presente la dimensión Campañas de comunicación de marketing coordinadas-. Este artículo describe un estudio exploratorio de 80 de las principales empresas públicas de Australia e investiga las relaciones entre la política percibida de la agencia, los conflictos de intereses y la orientación de CIM. Parte, además, de un estudio previo de Ewing, Bussy, y Ramaseshan (1998) donde ya se había evaluado esta problemática. 
Un tercer instrumento es el desarrollado por Low (2000), el cual se aplicó a una muestra de 421 empresas estadounidenses que habían implementado CIM. El estudio se hizo a través de la técnica de análisis de correlaciones bivariadas y regresión múltiple, y tuvo por objetivo la identificación de los factores que favorecen o dificultan la aplicación de la CIM, al igual que el análisis de los rendimientos de la organización en función de tal implementación. El autor propone la identificación de tres dimensiones -cada una identificada con un único ítem de evaluación-: la integración, la consistencia estratégica, y la consistencia del mensaje.

De acuerdo con Jiménez (2009), esta propuesta contiene bastantes limitaciones si se considera compleja la naturaleza multidimensional del concepto, que deja ver la falta de rigor en la conceptualización y la medición de las dimensiones propuestas.

Por último puede mencionarse la escala propuesta por de Lee y Park (2007), quienes a través de las técnicas de análisis factorial exploratorio y análisis de regresión establecieron una escala de medida para CIM, en donde identifican 4 dimensiones para el constructo: 1. Comunicaciones unificadas para un mensaje e imagen consistentes, 2. Comunicaciones diferenciadas para múltiples grupos de clientes; 3. Comunicaciones centradas en bases de datos para resultados tangibles; y 4. Relaciones que fomentan comunicaciones con clientes existentes. De acuerdo con Jiménez (2009), los procedimientos utilizados para desarrollar los 18 ítems de la escala final -cálculo del Alpha de Cronbach, validez de contenido, y validez convergente- ofrecen unos resultados satisfactorios en materia estadística, lo cual hace atractivo el instrumento para su aplicación. No obstante, cuenta con la limitación de que la muestra que se utilizó para su diseño estuvo restringida solo para Korea, lo cual podría influir en su proceso de validación empírica, y lleva a validarla en otros contextos.

\section{CONCLUSIONES}

Los aportes académicos presentados en este documento reflejan los resultados de más de 20 años de trabajo investigativo y propositivo por parte de varias escuelas de marketing que ven en la CIM una oportunidad novedosa para la aplicación del marketing a las organizaciones.

Como pudo verse en esta revisión, son muchas las visiones de los académicos frente a su conceptualización, que llevan a que paradójicamente hasta ahora se esté encontrando un camino común para el planteamiento de la definición. No obstante, ha sido tal el interés que ha suscitado en los académicos, que los ha exhortado a plantear no solo definiciones, sino también modelos, escenarios, y escalas de medición para evaluar su aplicación. 
Es por esto que a partir de este trabajo podría considerarse a CIM como uno de los enfoques de marketing con mayor potencial para explotar en el futuro de todos aquellos que se han desarrollado en los últimos tiempos. Incluso, al revisar las propuestas de aquellos que no ven en CIM más que un retoque de lo que ya se había planteado en relación con la comunicación de marketing, se sugiere la necesidad de pulir el enfoque y encontrar un rumbo mejor establecido en la teoría, y sobre todo, en la práctica de esta novedosa propuesta.

Desde este documento, se plantea CIM como una estrategia Integrada que puede tener importantes implicaciones en la gestión empresarial si sabe aprovecharse lo que hasta hoy se ha investigado y propuesto sobre ella. Es por esta razón, que se sugiere continuar con investigaciones como las que se han citado en este documento, pues esto ayudaría a comprender mejor este fenómeno y a conocer sus elementos más sobresalientes.

Esto sería de gran utilidad si se considera que CIM es un constructo relativamente nuevo, que en muchos contextos locales no ha tenido demasiadas aplicaciones, y que podría, por lo mismo, convertirse en un elemento diferenciador para las organizaciones que sepan aprenderlo y aplicarlo.

De otro lado, se plantea la necesidad de continuar con el desarrollo de investigaciones que permitan establecer un marco común para su aceptación desde todas las escuelas de marketing, pues aún se presentan serias limitaciones que se relacionan en especial con la posibilidad de evaluar técnica y financieramente su desempeño y sus contribuciones para la organización.

Además, se propone establecer un marco conceptual más riguroso con respecto a los beneficios que puede tener el sector empresarial con la aplicación de este nuevo enfoque de gestión de marketing, pues de este modo podría incentivarse el desarrollo de este concepto en las estrategias empresariales y en el desarrollo de marcas más fuertes.

Finalmente, se convoca a la evaluación de la CIM en relación con otros elementos y vertientes del marketing. Es así como se plantea la revisión de la relación entre la CIM y temas que hoy por hoy se incorporan para trabajar de manera conjunta para la gestión de marketing, tales como la gestión del valor de las marcas, los estudios de posicionamiento, y el desarrollo de actividades como el marketing digital, y el uso de nuevas tecnologías de información y telecomunicación. 


\section{BIBLIOGRAFIA}

AAAA - Asociación Americana de Agencias de Publicidad (AAAA por sus siglas en inglés) (1989). Integrated marketing Communications, Disponible en: http://www.aaaa.org/Pages/default. aspx [Consulta: 2012, abril 29]

Ambler, Tim, C. B. Bhattacharya, Julie Edell, Kevin Lane Keller, Katherine N. Lemon, y Vikas Mittal (2002), Relating Brand and Customer Perspectives on Marketing Management. En: Journal of Service Research, 5 (Agosto), 13-25.

Carlson L., Grove S., Dorsh M., (2003). Services advertising and Integrated Marketing Communications: An empirical Examination. En: Journal of Current Issues and Research in Advertising, Vol. 25 (2); pp. 69-82.

Chang, Y. y Thorson, E. (2004): Television and Web Advertising Synergies, En: Journal of Advertising, 33(2), pp. 75-84.

Cornelissen, J. P.; Lock, A. R. y Gardner, H. (2001). The organization of external communication disciplines: an integrative framework of dimensions and determinants. En: International Journal of Advertising, Vol. 20, n. 1 , pgs. 67-88.

Cornelissen, J. y Lock, A. (2000) Theoretical concept or management fashion. Examining the significance of IMC. En: Journal of Advertising Research, 40(5), pp. 7-15.

Cornwell, T. B. y Maignan, I. (1998): An International Review of Sponsorship Research. En: Journal of Advertising, 27(1), pp. 1-21.

Ducoffe, R. H., Sandler, D. y Secunda, E. (1996): A Survey of Senior Agency, Advertiser, and Media Executives on the Future of Advertising. En: Journal of Current Issues and Research in Advertising, 18(1), pp. 1-19.

Duncan, T., y Everett, S. (1993). Client Perceptions of Integrated Marketing Communications. En: Journal of Advertising Research, May/Jun93, Vol. 33, Issue 3. pp. 30-39.

Duncan, T. y Caywood C. (1996). The concept, process, and evolution of integrated marketing communication. En: Thorson, E. y Moore, J. (eds) Integrated Communication: Synergy of Persuasive Voices. Mahwah, NJ: Lawrence Erlbaum, pp. 13-34.

Duncan, T. R. y Moriarty, S. E. (1997). Driving brand value. Using integrated marketing to manage profitable stakeholder relationships. New York: McGraw-Hill. 284 pp.

Duncan, T. (2002). IMC: Using Advertising and Promotion to Build Brands. International Edition, The McGraw-Hill Companies. 783 pp.

Duncan, T.; Mulhern, F. (2004), A white paper on the status, scope and future of IMC. IMC Symposium co-sponsored by IMC programs at Northwestern University and University of Denver (marzo). 
Ewing, M.T., De Bussy, N.M. and Ramaseshan, B. (1998) Integrated marketing communications: Conflicts of interest, politics and performance. En: J. B Ford and E.D. Homeycutt Jr. (eds) Developments in Marketing Science. pp. 265-72.

Ewing, M. T., De Bussy, N. M. y Caruana, A. (2000). Perceived agency politics and conflicts of interest as potential barriers to IMC orientation. En: Journal of Marketing Communications, 6 (2), pp. 107-119.

Fortini-Campbell, L. (1992). Hitting the Sweet Spot: How consumer insights can inspire better marketing and advertising. Chicago. IL: Copy Workshop. 246 pp.

Garreston, J. y Burton, S. (2005). The role of spokescharacters as advertisement and package cues in integrated marketing communications. En: Journal of Marketing, 69 (4), pp.118-132.

Gopalakrishna, S. y Chatterjee R. (1992). A communications response model for a mature industrial product. En: Journal of Marketing Research, 29(2), pp.189-200.

Gould, S. J. (2000). The State of IMC Research and Applications. En: Journal of Advertising Research 40, 5 pp. 22-23.

Grove, S., Carlson, L., y Dorsh, M. (2007). Comparing the application of Integrated Marketing Communication (IMC) in magazine ads across product type and time. En: Journal of Advertising, 36(1), pp. 37-54.

Hartley, B. y Pickton, D. (1999). Integrated Marketing Communications require a New way of thinking. En: Journal of marketing Communications, 5(2): pp. 97-106.

Homburg C.; Workman, Jr. J.P., y Kromer, H. (1999). Marketing's influence within the firm. En: Journal of Marketing, 63(2), pp. 1-17.

Hutton, J. G. (1996), Integrated Marketing Communications and Evolution of Marketing Thought. En: Journal of Business Research, Vol. 37, pp.139-146.

Jiménez D., Sánchez M., Gázquez J. (2006). An exploratory study on IMC in Spanish communication agencies: control decisions, barriers, benefits, and its future. International Marketing Trends Conference, Università Ca' Foscari Venezia, Venecia, Italia. 20 y 21 de enero.

Jiménez D. (2009). La integración de la Comunicación Interna de Marketing y su uso en la gestión del Conocimiento del Mercado. Tesis doctoral. Departamento de Dirección y Gestión de Empresas, Universidad de Almería, España.

Jiménez D. (2007). La comunicación integral de Marketing: Análisis del fenómeno desde una perspectiva Teórico-Práctica. En: Revista Investigación y Marketing.-Asociación Española de Estudios de Mercado, Marketing y Opinión, N. ${ }^{\circ}$ 90. Pp. 12-18.

Jin, H.S.; (2003-04). Compounding consumer interest: Effects of advertising campaign publicity on the ability to recall subsequent advertisements. En: Journal os Advertising, 32 (4), pp. 29-41 
Keegan, W., Moriarty, S. y Duncan, T. (1992). Marketing. Prentice-Hall, Englewood Cliffs, NJ. 743 pp.

Keller. K. L. (2001) Mastering the Marketing Communications Mix: Micro and Macro Perspectives on Integrated Marketing Communication Programs. En: Journal of Marketing Management, 17, Pp. 819-847.

Kerr G., Schultz D., Patti Ch., Kim I. (2008). An inside-out approach to integrated marketing communication, An international analysis. En: International Journal of Advertising, 27(4), pp. $511-548$

Kim I., Han D. y Schultz D. (2004), Understanding the Diffusion of Integrated Marketing Communications. En: Journal of Advertising Research. Marzo pp. 31-45

Kitchen, P. y Schultz, D. (1999) A Multi-Country Comparison of the Drive for IMC. En: Journal of Advertising Research, 39 (1), pp.21-38.

Kitchen, P. J.; Brignell, J.; Li, T. y Jones, G. S. (2004): The Emergence of IMC: A Theoretical Perspective. En: Journal of Advertising Research, 44(1), pp. 19-30.

Kliatchko, J. (2005). Towards a New Definition of Integrated Marketing Communications (IMC). En: International Journal of Advertising 24(1) pp. 7-34.

Kliatchko, J. (2008). Revisiting the IMC construct- A revised definition and four pillars. En: International Journal of Advertising, 27(1), pp. 133-160

Kotler, P., Armstrong, G., Saunders, J. y Wong, V. (1999). Principles of Marketing. (2nd European edition). London: Prentice Hall Europe. 1056 pp.

Lee D. y Park C. (2007). Conceptualization and measurement of multidimensionality of Integrated Marketing Communications. En: Journal of Advertising Research, 47(3), pp. 222-236.

Low, G. S. (2000). Correlates of integrated marketing communications. En: Journal of Advertising Research, 40 (3), 27-39.

Madhavaram S, Badrinarayanan V., y McDonald R. (2005). Integrated Marketing Communication (IMC) and Brand Identity as Critical Components of Brand Equity Strategy. En: Journal of Advertising, vol. 34, n. ${ }^{\circ}$, pp. 69-80

Moriarty, S. E. (1994): PR and IMC: The Benefits of Integration, Public Relations Quarterly, 39(3), pp. 38-44.

Naik, P. A. y Raman, K. (2003): Understanding the Impact of Synergy in Multimedia Communications. En: Journal of Marketing Research, 40(4), pp. 375-388.

Navarro M. A. (2008). La integración de la comunicación: Análisis de su implicación para el consumidor y el capital de marca. Tesis doctoral. Universidad de Murcia.

Nowak, G. y Phelps, J. (1994) Conceptualizing the integrated marketing communications' phenomenon: an examination of its impact on advertising practices and its implications for advertising research. En: Journal of Current Issues and Research in Advertising, 16(1), pp. 49-66.

Percy, L.; Rossiter, J. R.; Elliot, R. (2001), Strategic Advertising Management, New York, Oxford University Press Inc. En: Kim, I.; Han, D.; Schultz, D. E. (2004), Understanding the Diffusion 
Comunicación integrada de marketing: un acercamiento a la evolución del concepto

of Integrated Marketing Communication, Journal of Advertising Research, Vol. 44, March, pp. 31-45.

Phelps J. y Jhonson E. (1996) Entering the quagmire: Examining the meaning of Integrated Marketing Communications. En: Journal of Marketing Communications. 2, pp. 159-172.

Pickton D. y Hartley R. (1998). Measuring Integration: An assessment of the quality of Integrated Marketing Communications. En: International Journal of Advertising 17:447-465.

Porcú L. y Barrio S. (2008), Modelización de los antecedentes y consecuentes de la comunicación integrada de marketing. Trabajo Fin de Máster, Programa de Doctorado en Técnicas Avanzadas de Planificación y Gestión Comercial: Marketing y Consumo, Departamento de Comercialización e Investigación de Mercados, Universidad de Granada, Granada, España.

Reid, M. (2005). Performance auditing of integrated marketing communications (IMC) actions and outcomes. En: Journal of Advertising, 34 (4), 41-54.

Reid, M.; Luxton, S.; Mavondo, F. (2005), The relationship between Integrated Marketing Communication, Market Orientation and Brand orientation. En: Journal of Advertising, Vol. 34, N. ${ }^{\circ} 4$, pp. 11-23.

Rust, R. T.; Ambler, T.; Carpenter, G. S.; Kumar, V.; Srivastava, R. K. (2004), Measuring Marketing Productivity: Current Knowledge and Future Directions. En: Journal of Marketing, Vol. 68, Octubre, pp. 76-89.

Schultz, D.E. (1991). Integrated Marketing Communications: The Status of Integrated Marketing Communications Programs in the US. En: Journal of Promotion Management, 1: 37-41.

Schultz, D.E. (1997) The evolving nature of integrated communications. En: Journal of Integrated Communications (1997-1998 edition), pp. 11-18.

Schultz, D. (1998) Determining how brand communication works in the short and long terms, International. En: Journal of Advertising, 17(4), pp. 403-426.

Schultz, D. E.; Cole, B.; Bailey, S. (2004), Implementing the 'Connect the Dots' Approach to Marketing Communication. En: International Journal of Advertising, N. 23, pp. 455-477.

Schultz, D. E.; y Kitchen, P. (2000). A response theoretical concept or management fashion? En: Journal of Advertising Research, 40(5): 17-21

Schultz D. E., y Patti C. (2009). The evolution of IMC: IMC in a Customer-driven Marketplace. En: Journal of Marketing Communications. Vol. 15, Nos. 2-3, April-July 2009, 75-84

Schultz, D. E. y Schultz, H. F. (1998) Transitioning marketing communication into the twenty-first century. En: Journal of Marketing Communications, 4(1), pp. 9-26.

Schultz D. E. y Schultz H. (2004). IMC The next generation. Five steps for delivering value and measuring returns using marketing communications. McGraw-Hill Nueva York. 320 pp.

Schultz, D. E., Tannenbaum S. y Lauterbom R., (1993) The New Marketing Paradigm, Integrated marketing Communications, NTC publishing Group.

Schultz, D.E. y Walters, J. (1997) Measuring Brand Communication ROI. New York: Association of National Advertisers. 
Schumann, D., Dyer, B.; y Pecktus, E. (1996). The vulnerability of Integrated marketing communication: The ptencial for boomerang effects, en E. Thorson y J. Moore (eds.); Integrated Communication: Synergy of Persuasive voices, pp. 51-64, Lawrence Erlbaum Associates, Mahwah, NJ.

Shimp T. (2000). Advertising Promotion: Supplemental Aspects of Integrated Marketing Communications.5th ed. Fort Worth, TX: The Dryden Press, Harcourt College Publishers. $650 \mathrm{pp}$.

Shimp T. (2003). Advertising Promotion: Supplemental Aspects of Integrated Marketing Communications, $6^{\mathrm{a}}$ ed. Thomson South-Western, Mason, Ohio.752 p.

Smith, T. M.; Gopalakrishna, S. y Smith, P. M. (2004): The Complementary Effect of Trade Shows on Personal Selling, En: International Journal of Research in Marketing, Vol. 21(1), pp. 61-76.

Spotts, H.E., Lambert, D.R. y Joyce, M.L. (1998) Marketing déjà vu: the discovery of integrated marketing communications. En: Journal of Marketing Education, 20(3), pp. 210-218.

Swain, W.N. (2004). Perceptions of IMC after a decade of development: Who's at the wheel, and how can we measure success? En: Journal of Advertising Research, 44(1), pp. 46-55.

Workman, Jr. J.P; Homburg, C., y Gruner, K. (1998). Marketing Organizations: An integrative frameworkof dimensions and determinants. En: Journal of Marketing, 62(3), pp. 21-41. 